FORMATION Formation emploi

Revue française de sciences sociales

106 | avril-juin 2009

Pêle-mêle

\title{
Lecture et écriture au travail : les enjeux en termes de formation et de conditions de travail
}

Lesen und Schreiben am Arbeitsplatz: Herausforderungen in Bezug auf Ausbildung und Arbeitsbedingungen

Reading and writing in the workplace: the stakes in terms of training and working conditions

Lectura y escritura en el trabajo : desafíos en términos de formación y de condiciones de trabajo

Frédéric Moatty et Françoise Rouard

\section{OpenEdition}

Journals

Édition électronique

URL : http://journals.openedition.org/formationemploi/1943

DOI : 10.4000/formationemploi.1943

ISSN : 2107-0946

Éditeur

La Documentation française

Édition imprimée

Date de publication : 1 juin 2009

Pagination : 59-73

ISSN : 0759-6340

Référence électronique

Frédéric Moatty et Françoise Rouard, «Lecture et écriture au travail : les enjeux en termes de formation et de conditions de travail », Formation emploi [En ligne], 106 | avril-juin 2009, mis en ligne le 01 juin 2011, consulté le 30 octobre 2020. URL : http://journals.openedition.org/formationemploi/ 1943 ; DOI : https://doi.org/10.4000/formationemploi.1943

(C) Tous droits réservés 


\title{
Lecture et écriture au travail : les enjeux en termes de formation et de conditions de travail
}

Frédéric Moatty et Françoise Rouard*

\begin{abstract}
Au travail, les activités liées à l'écrit, l'écriture mais aussi la lecture professionnelles, sont de plus en plus présentes. Aussi, la formation des salariés aux compétences langagières au travail concerne tous les salariés et ne se réduit pas à un problème de contenus de formation; elle nécessite aussi de prendre en compte les conditions du travail intellectuel dans les entreprises.
\end{abstract}

Le propos de l'article est de montrer l'importance des activités de lecture et d'écriture au travail ainsi que les problèmes spécifiques de formation qui leur sont liés. L'enjeu est de réfléchir à l'adaptation des formations des salariés mais aussi à celle des conditions du travail intellectuel et de son organisation.

La formation générale au travail est généralement mesurée à l'aune du niveau scolaire ou de la possession d'un diplôme, ce qui est approprié si la formation est en relation avec le poste occupé. Mais au travail, il existe souvent un décalage entre le titre obtenu et le poste, et ce décalage s'accentue souvent avec l'âge, les transformations des métiers, les changements organisationnels et technologiques. Par ailleurs, l'activité de travail conduit à mobiliser de manière variée des compétences générales; dès lors, il semble pertinent d'identifier et de mesurer la formation générale au moment de sa mise en œuvre. Deux activités liées à l'écrit, la lecture et l'écriture professionnelles, peuvent en constituer des indicateurs généraux.
La pertinence de ces indicateurs est renforcée par l'expansion des nouveaux outils liés aux Technologies de l'information et de la communication (TIC)

* Frédéric Moatty est sociologue du travail, chargé de recherche au Centre d'études de l'emploi-Tepp - Travail, emploi, politiques publiques (Fédération de Recherche n ${ }^{\circ} 3126$-CNRS). Ses domaines d'investigation sont le travail et l'emploi, les technologies de l'information et de la communication (TIC), l'écrit au travail, le changement organisationnel, le travail collectif. II a notamment publié : Greenan N., Guillemot D., Moatty F. (2005) "Comment les relations au travail sont-elles mesurées dans l'enquête Changements Organisationnels et Informatisation? ", Réseaux, vol. 23, n 134, décembre, pp. 21-63. Moatty F., Rouard F., Teiger C. (2005), "Montée de l'écrit au travail et transformation de l'évaluation", in M.-C. Bureau et E. Marchal (Dir.), Au risque de l'évaluation. Salariés et candidats à l'emploi soumis aux aléas du jugement, Villeneuve d'Asca, Presses Universitaires du Septentrion, pp. 175-201. 
Françoise Rouard est linguiste, maître de conférences au Conservatoire national des arts et métiers /Cnam Paris, chercheure au Laboratoire interdisciplinaire de sociologie économique (Lise/CNRS/CNAM, UMR unité mixte de recherche 5262). Ses domaines d'investigation sont : langage et travail, écrit et oral au travail, écrit dans l'organisation du travail, écrit et Technologies de l'information et de la communication, compétences linguistiques langagières et culturelles. Elle a notamment publié : Rouard F. (1995), "L'individu et ses traces dans les écrits au travail ", Le Gré des langues n ${ }^{\circ}$ 9, L'Harmattan, Paris, pp. 152-175. Peluau B., Rouard F. (2007), "Rôle et enjeux de la formation continue au langage dans la professionnalisation des travailleurs sociaux ", Vie sociale, Dossier « Voix des précaires et langage du social » $n^{\circ} 3$, pp. $111-121$.

au travail, qui exacerbe les problèmes liés à l'écrit et à sa maîtrise. Mais le primat accordé à la technique dans les formations aux TIC complique l'identification des difficultés et des besoins de formation générale.

L'article est organisé en trois parties. La première rappelle les travaux sur la place et l'importance du langage au travail dans les services comme dans les activités de production industrielle. Elle s'intéresse aux enjeux de formation et aux compétences langagières à l'œuvre au travail, notamment dans les activités liées à l'écrit, la lecture et l'écriture. La seconde partie présente les résultats d'une enquête préalable auprès de techniciens et d'ingénieurs en formation au CNAM (Conservatoire national des arts et métiers); elle vise à élaborer les catégories pertinentes pour interroger les salariés sur leurs activités de lecture/ écriture. Cette enquête a permis d'inventorier ces activités, de préciser leurs propriétés et de montrer l'importance des conditions de leur réalisation. Elle a mis en évidence les problèmes de formation et la dimension du temps, dans la mesure où ces activités sont chronophages et parfois reportées hors travail. La troisième partie mesure différentes catégories d'activités de lecture/écriture dans une population spécifique, les actifs dont la durée effective de travail dépasse la durée de travail contractuelle (dépassements horaires), toutes catégories professionnelles confondues. Elle montre l'importance de ces activités lors des dépassements horaires et leurs clivages selon le niveau scolaire, les professions, le genre et les conditions de travail. La conclusion met en perspective les résultats, souligne les manques de formation à ces activités et montre que les conditions de travail adéquates ne sont pas toujours réunies, avec la sous-estimation de ces activités et le report des contraintes de réalisation sur les individus.

\section{LES COMPÉTENCES LANGAGIÈRES DANS L'ÉCRIT AU TRAVAIL}

Les recherches sur le langage au travail commencent, en France, dans les années $90^{1}$. Nous en rappelons les principaux éléments, nous analysons ensuite les enjeux de formation qui sont associés à l'importance du langage dans le travail ainsi que les compétences à l'œuvre dans les activités langagières.

\section{La dimension langagière du travail}

Analyser l'univers du travail par l'entrée du langage, tel est le propos des chercheurs réunis dans le réseau interdisciplinaire «Langage et Travail » du CNRS. Leur objectif était de décrire les pratiques et les systèmes langagiers et communicationnels (écrits, oraux et graphiques) afin d'appréhender l'activité de travail des opérateurs au sein des organisations. L'activité de langage au travail (Boutet, 1995 ; Boutet, Gardin, 2001 ; Boutet, 2008) a été étudiée au sein «d'agencements organisationnels 》(Girin, 1995). La relation du langage au travail ne va pas de soi : la parole soutient l'activité, elle peut la gêner, elle peut être enfin l'activité elle-même (Teiger, 1995). Ultérieurement, les travaux se sont intéressés au lien entre communication, cognition et action au travail (Borzeix, Fraenkel, 2001).

\section{Les enjeux de formation}

Les enjeux de formation du langage au travail ont fait l'objet d'un colloque organisé, en 1998, conjointement par le CNAM, l'INRP (Institut national de

\footnotetext{
${ }^{1}$ Colloques : 1989, « Travail et pratiques langagières », Paris, 2425 avril ; 1998, "Le langage dans les restructurations du travail. Paradoxes et enjeux », Paris, Inetop, 24-26 septembre; 1998, "Langage(s) et travail : enjeux de formation ", INRP/CNAM/ CNRS-LT, Paris, 13-15 octobre.
} 


\section{Encadré 1 \\ Une diversité d'approches sur l'écrit au travail}

Dans le champ "Langage et Travail», la montée de l'écrit au travail a conduit des chercheurs de différentes disciplines à s'interroger sur les compétences langagières à l'œuvre dans l'écrit (Rouard, 1995, 1998), sur l'écrit dans sa relation avec l'oral (Grosjean, Lacoste 1998), sur le passage d'instructions orales à des instructions écrites (Moatty, 1995), sur la mise en place de la traçabilité (Fraenkel, 1995), le langage dans les organisations (Pène, Borzeix, Fraenkel, 2001), les normes de qualité (Cochoy, Garel, Terssac, 1998), les usages professionnels des écrits électroniques (Bailly et alii, 2002) ou sur les temps de l'écrit, les procédures managériales d'évaluation et les pratiques de lecture à l'heure du numérique (Moatty, Rouard, Teiger, 2004, 2005 , 2007).

recherche pédagogique) et le réseau CNRS « Langage et Travail ». Ce colloque a relié l'approche théorique pluridisciplinaire du langage au travail et les approches formatives en formation initiale et permanente dans l'enseignement technique secondaire et supérieur. Les communications présentées concernaient majoritairement la dimension didactique, soit le choix des contenus d'enseignement et la formation des enseignants. D'autres ont analysé les pratiques professionnelles au travail, notamment langagières, pour en dégager des besoins voire des dispositifs de formation. L'accent a principalement été mis sur les apprentissages (lexique de spécialités, langages techniques...), les univers sémiotiques (dessins, paroles, écritures), les dispositifs de formation spécifiques (éducateurs, infirmiers, mécaniciens...) et les référentiels (programmes et manuels). Parallèlement à cette approche catégorielle, la question de la formation générale a été abordée dans sa relation avec la formation technique ou professionnelle en termes de compétences de base, de connaissance des langues, de compétences générales (parole, lecture, écriture). Nous proposons d'approfondir cette question au regard des activités de lectureécriture développées au travail.

\section{L'écrit au travail : des compétences diverses et un manque de formation}

La question des compétences a suscité de nombreux débats, en lien avec les qualifications, que nous ne développerons pas ici (Dugué, 1994). De même, la question du manque de formation se pose, puisque $39 \%$ des salariés la ressentent comme une gêne dans l'exercice de leur activité (Pommier, Zamora, 2008). Nous étudions les compétences à l'œuvre dans l'écrit au travail. Il s'agit de compétences générales - linguistiques et techniques -, culturelles, ou encore sociales dans leur dimension relationnelle (Rouard, 1998). L'ensemble de ces trois types de compétences relève d'une articulation entre un capital langagier et culturel et un capital social.

Les compétences générales linguistiques se rapportent à la maîtrise des langues orales et écrites. Les compétences générales techniques renvoient à l'acquisition des techniques rédactionnelles, de prise de parole et de lecture. Les compétences culturelles renvoient à la connaissance des genres d'écrits, des styles d'écriture et des supports, du point de vue de leur histoire et de leurs usages ${ }^{2}$, sans oublier la culture de l'écrit propre à un secteur ou à une entreprise. Les compétences sociales renvoient à la dimension relationnelle des activités d'écriture, c'est-à-dire la prise en compte du contexte de production, d'utilisation et de circulation des documents. Elles supposent de savoir se situer face à autrui, pour évaluer le niveau de synthèse, le style d'écriture et le choix d'un support adaptés à l'interaction. Elles impliquent de distinguer «écrits pour soi»- brouillons ou écrits intermédiaires à destination d'un groupe de pairs - et «écrits pour autrui» (Moatty, Rouard, Teiger, 2004)formalisés, c'est-à-dire achevés et validés dans le respect des règles en usage, notamment les niveaux de confidentialité - afin de maîtriser la circulation des documents en termes de respect ou de contournement des règles. Elles nécessitent également la maîtrise de la production des documents qui peut

2 Sur ce point, rappelons les travaux de Chartier sur la culture de l'écrit, et notamment ceux sur la correspondance (Chartier, 1992). 
différencier les rédacteurs des signataires. Elles supposent enfin, en cas de besoin, de pouvoir mobiliser un réseau d'aide professionnel, amical ou familial.

Si l'école forme à des degrés divers aux compétences générales, linguistiques et techniques, elle ne forme pas aux compétences sociales d'écriture. Ce problème de formation est accentué par le fait qu'au travail, les compétences d'écriture et de lecture sont considérées comme allant de soi, relevant des acquisitions scolaires initiales, alors que les écrits de travail obéissent à d'autres normes et mobilisent d'autres savoir-faire tels que le choix d'un vocabulaire adapté, l'aptitude à la synthèse, le degré de maîtrise de la situation et des temps de réalisation. Prenons l'exemple du rapport d'intervention d'un technicien de maintenance informatique, rédigé suite à la résolution d'une panne chez un client (Rouard, 1995). Dans cet écrit, à double destinataire - le client et la hiérarchie du technicien - le technicien doit montrer une double expertise technique et relationnelle. La restitution écrite de son activité «doit faire signe» sur son professionnalisme (le technicien a effectué son travail selon les normes de performance et d'objectifs propres à son univers de travail), mais en plus, elle « fait trace » et vaut enregistrement pour lui et ses actes (son écrit doit protéger l'entreprise d'un éventuel litige avec le client). En écrivant, le technicien prend donc des risques, avec lesquels il dit devoir « se débrouiller pour durer » dans son activité. L'écriture professionnelle est une activité à risques qui suppose d'intégrer les normes en usage dans l'univers professionnel et d'être formé à l'art et la manière de présenter un réel composé d'actions et de faits qui associent soi et les autres.

La prise de conscience des difficultés d'acquisition de ces compétences a conduit la plupart des formations techniques à visée professionnelle à inclure des enseignements de communication ${ }^{3}$. Il s'agit d'enseignements de culture générale destinés à développer des compétences langagières, tant à l'oral qu'à l'écrit, et des compétences relationnelles, notamment en termes de place et de bonne distance.

\footnotetext{
${ }^{3}$ Le CNAM a ainsi créé un enseignement de communication pour les élèves ingénieurs, $c f$. "Approches de l'écrit», cahier $n^{\circ} 1$, Communication/Culture/Expression (CCE-CNAM), 1998.
}

\section{CONSTRUIRE DES CATÉGORIES D'ANALYSE SUR LES PRATIQUES D'ÉCRITURE}

Les recherches sur les activités d'écriture en tant qu'activités professionnelles ordinaires, notamment celles du réseau «Langage et Travail », s'étaient peu intéressées à leurs conditions de réalisation, c'est-àdire aux conditions de travail qui y sont liées; elles traitaient peu des activités de lecture. Par ailleurs, ces recherches se caractérisent par leur caractère qualitatif tandis que les enquêtes quantitatives sur le travail ont, jusqu'à très récemment, peu abordé les activités de lecture/écriture ${ }^{4}$.

Nous avons élaboré un dispositif de recherche visant à construire des catégories pertinentes d'interrogation des activités de lecture/écriture grâce à une enquête qualitative puis à les mesurer quantitativement et à les relier aux conditions de travail; il s'agissait de contribuer à leur prise en compte dans les enquêtes ultérieures. Cette partie développe des aspects qualitatifs de cette recherche et la suivante ses aspects quantitatifs.

L'enquête qualitative a choisi d'étudier une population en transition, les techniciens en formation d'ingénieur au CNAM, qui exercent des activités productives et réalisent des tâches quotidiennes d'écriture et de lecture. Cette population est engagée dans un cursus de formation avec un projet de mobilité professionnelle (Grignon, 1976; Pottier, 1996) vers le groupe cadre, ce qui suppose une trajectoire culturelle et l'acquisition de compétences langagières. Cet univers technique articule culture professionnelle, culture technique et culture générale.

Une première enquête exploratoire ( $c f$. encadré 2) a construit un cadre d'analyse pour l'élaboration des catégories liées aux activités de lecture et d'écriture professionnelles et leur inventaire. Nous avons étudié les façons de lire, d'écrire et de dire dans l'activité productive. Cette approche des activités et des pratiques langagières des salariés s'est déroulée dans un contexte interdisciplinaire micro et macro, articulant ergonomie, linguistique et sociologie.

${ }^{4}$ L'enquête «Conditions de Travail 2005 », réalisée par la DARES, est la première à interroger les activités de lecture/ écriture qu'elle mesure en termes de temps journalier. 


\section{Encadré 2}

\section{L'enquête exploratoire : « La recherche sur des techniciens en formation d'ingénieurs, en transition professionnelle, et leurs pratiques d'écriture au travail »}

Cette étude exploratoire interdisciplinaire (linguistique, ergonomie, sociologie) a été menée de 2000 à 2002 avec de petits groupes de techniciens issus de divers secteurs de l'industrie et des services alors en cours de formation permanente d'ingénieur au CNAM (Conservatoire national des arts et métiers). Elle visait à mettre en relation les évolutions récentes du travail avec les pratiques langagières liées à l'écrit, manifestées dans le travail de ces salariés pour qui la tentative de transition professionnelle doit s'accompagner d'une transition culturelle liée à la maîtrise de nouveaux codes sociaux.

Cette situation de transition révèle les différences de nature qualitative entre une activité principalement basée sur des compétences techniques et un travail de cadre requérant, outre les compétences techniques, des compétences scripturales, culturelles et relationnelles. Ces techniciens vivent des mutations sur tous les plans de leur vie professionnelle, et notamment dans leur rapport au langage écrit lécriture et lecture). Ce qui génère des pratiques langagières nouvelles requérant l'acquisition de compétences inhabituelles pour des personnes issues de formations initiales techniques traditionnelles.

L'objectif immédiat était d'inventorier et de catégoriser l'écriture et la lecture dans leur travail dans ses dimensions formelles, fonctionnelles et culturelles. L'objectif à plus long terme était de construire de façon inductive les éléments d'une enquête extensive sur ce type de pratiques professionnelles. La démarche a consisté à pratiquer une analyse du travail individuelle et collective à distance, en construisant une situation de recherche participative visant à faire produire progressivement par ces techniciens en formation des données concernant leurs activités liées à l'écrit en situation de travail ainsi que leurs commentaires, leurs représentations et leur construction "spontanée » de la catégorisation de ces écrits.

Cette démarche de co-construction progressive des catégories et du questionnaire s'est déroulée en trois phases : un échantillon restreint de 4 techniciens élèves ingénieurs pour une étude clinique des pratiques de lecture et d'écriture professionnelle débouchant sur l'élaboration de catégories partagées, un échantillon plus large composé de 36 techniciens pour la construction d'un questionnaire, enfin un groupe de 23 personnes plus diversifié du point de vue professionnel afin de valider le questionnaire. La méthodologie de cette recherche interdisciplinaire a donc consisté en une approche inductive, qualitative et systématisée préalable à des enquêtes quantitatives. II s'agissait d'élaborer les dimensions pertinentes pour une enquête sur le thème de l'écrit au travail auprès de leurs pairs ainsi qu'une ébauche de questionnaire, analyser les réponses à ces mini-questionnaires, discuter collectivement les exercices individuels. Recueillir des traces de l'écriture au travail des techniciens enquêtés a nécessité plusieurs séances de travail afin de répertorier l'ensemble des thèmes : les "écrits 》 professionnels, les pratiques de lecture ou d'écriture, les représentations associées et la catégorisation des écrits, les conditions de travail et les difficultés rencontrées (Teiger, Rouard, Moatty, 2000, 2002).

À l'issue de cette étude exploratoire, un questionnaire a été réalisé et diffusé auprès de 501 visiteurs, techniciens et non techniciens, sur le forum d'inscription du CNAM, sur le thème des pratiques de lecture et d'écriture en fonction des supports papier et électronique.

Ce cadre d'analyse a permis l'élaboration d'un questionnaire auprès d'une population plus large de nouveaux «visiteurs », c'est-à-dire de candidats à l'inscription au CNAM, ingénieurs et techniciens. Les résultats de ces enquêtes peuvent être présentés de manière synthétique (Teiger, Rouard, Moatty,
2000, 2002 ; Moatty, Rouard, Teiger, 2004, 2005). Nous les exposons sous trois angles: les propriétés des activités de lecture/écriture au travail, la spécificité des compétences langagières à l'œuvre dans l'écrit, et les apprentissages par «essais-erreurs » et leurs risques. 


\section{Les dix propriétés des activités de lecture/écriture au travail}

L'écrit, au regard de l'oral, a trois propriétés essentielles : visibilité, permanence et fixité (Christin, 2001). Au travail, l'écrit et l'oral sont imbriqués et l'écrit assume des fonctions spécifiques : il fait autorité en tant que trace, est un support d'enregistrement et de mémoire, un outil de coordination des temps et des acteurs... Il est enfin un outil de travail avec les autres et pour les individus. On observe une " mise à l'écrit » généralisée dans le monde professionnel qui peut conduire à une "insécurité linguistique $»^{6}$ (Rouard, 2000) ou à une exclusion sur le marché du travail pour les bas niveaux de qualification (Moatty, 2000).

Au-delà de l'importance des activités de lecture/ écriture au travail, dix propriétés se dégagent :

- L'importance quotidienne prise par les activités de lecture et d'écriture au travail ;

- Ces activités sont imbriquées : au travail on ne lit pas pour lire, mais on lit avant d'écrire, pour écrire, ou encore pour valider un document ;

- Les écrits (lus ou produits) au travail sont situés, comme en témoigne la spécificité des désignations qui renvoient par exemple à leur circulation (« document client-fournisseur $»)$, ou à leur support (papier comme le « post-it » ou électronique comme « le courriel ») ;

- L'activité d'écriture et encore plus celle de lecture apparaissent comme invisibles au travail, contrairement aux écrits réalisés car elles sont souvent désignées par la finalité du travail. «Faire de la veille technologique », par exemple, suppose une activité de recherche d'information, d'archivage, de lecture, d'annotation et de synthèse ;

- Ces activités sont consommatrices de temps, y compris au niveau journalier; souvent elles prennent plus de temps que prévu ;

- Elles se surajoutent à l'activité et elles s'effectuent sous contrainte de pression temporelle;

- Ces activités chronophages ne sont pas toujours considérées comme du travail par les collègues ou par la hiérarchie. Par exemple, lire est perçu comme

${ }^{5} \mathrm{La}$ forme écrite rend visible, la permanence du support permet la trace dans le temps et dans l'espace et la fixité du code linguistique permet l'échange.

${ }^{6}$ La notion d'insécurité linguistique renvoie à une maîtrise insuffisante des normes qui fragilise les salariés sur le plan professionnel, identitaire et social. de l'inoccupation dans le monde de la production, pour un salarié qui effectue une activité de veille technologique dans une entreprise de pointe ;

- L'écriture et surtout la lecture peuvent être déportées en partie dans le hors travail, que ce soit à domicile ou au travail pendant les heures supplémentaires ;

- La lecture et l'écriture au travail sont souvent des activités « outillées et équipées » (crayons, stabilo, TIC) ;

- Enfin, la lecture et l'écriture au travail sont clivées socialement, par exemple selon les qualifications professionnelles ou les diplômes.

\section{Spécificité des compétences langagières en jeu dans l'écrit}

Les manuels distinguent traditionnellement deux grands genres d'écrits professionnels: le compterendu et le rapport. Le compte-rendu, écrit de type factuel, nécessite des savoirs implicites d'analyse, d'écoute et de synthèse pour "aller à l'essentiel », d'euphémisation stylistique pour «dire sans dire». Le rédacteur doit maîtriser « la bonne distance », les enjeux relationnels et la culture écrite de son entreprise. Il doit identifier le statut des personnes, participants ou destinataires, les enjeux de la situation d'écriture, pour produire une synthèse adaptée.

Par différence, le rapport mobilise les opinions, les valeurs, les points de vue et les choix personnels. Argumenter suppose de prendre position sur des faits (objets, situations, interprétations, acteurs...) à partir de systèmes de valeurs. Les "rapports », écrits de type modal, c'est-à-dire qui engagent la subjectivité et l'imaginaire du rédacteur, font partie des « écrits à risques », souvent rédigés par les techniciens au-delà de leur temps de travail contractuel. L'argumentation comporte des risques qui varient selon que la place occupée par le rédacteur l'autorise et le légitime ou non à prendre la plume. En tant qu'expert, le technicien doit manier la rhétorique pour faire valoir sa position sans s'engager dans la prise de décision.

\section{Les apprentissages:}

" essais-erreurs » et risques

$\mathrm{Au}$ travail, l'apprentissage des règles d'usage, de production et de mise en circulation des documents professionnels, avec validation et signature, se fait 
par « essais et erreurs » et sous contrainte. Ce type d'apprentissage est très insécurisant et insatisfaisant, car porteur de risques ou même d'humiliations. Par exemple, à propos de l'élaboration de dossiers techniques, dans le cadre d'une démarche qualité, l'objectif de productivité de l'entreprise lié à la pression du temps et à la complexité des questions traitées s'oppose à des objectifs de formalisation en relation à des fonctions de mémoire ou de preuve de l'activité. Pour gagner du temps, les techniciens contournent les règles de la procédure officielle de circulation des documents, dans certaines étapes du processus, et ne formalisent leur production écrite qu'une fois tous les problèmes techniques réglés officieusement entre pairs par différentes voies (usage d'un fax, d'un courrier électronique ou du téléphone, etc.) (Teiger, Rouard, Moatty, 2000). L'usage d'un document inabouti, d'un brouillon, qui ne respecte pas les normes et consignes d'écriture dans un dossier officiel, est sanctionné par la hiérarchie.

Enfin, dans les apprentissages scolaires, les activités de lecture et d'écriture sont distinctes, les temps et moments d'apprentissage sont différents; or dans l'univers du travail, cette séparation est discutable. Ces deux activités peuvent être simultanées : pour les techniciens enquêtés, lire un document peut signifier l'annoter. Relire un document peut signifier le valider et donc éventuellement le signer, le contresigner ou écrire des modifications.

\section{Lecture/écriture à l'ère du numérique}

Dans un contexte marqué par l'usage des TIC, la question des apprentissages de l'écrit est reposée au regard des nouveaux outils. Au-delà des apprentissages purement techniques du maniement des nouveaux outils, les compétences générales d'écriture au travail sontelles modifiées substantiellement? Une analyse croisant les usages des principaux documents professionnels selon les supports papier ou électronique, auprès d'une population de 501 nouveaux visiteurs du CNAM (Moatty, Rouard, Teiger, 2007), révèle que les documents ne s'opposent pas radicalement en fonction de leur support mais se différencient en premier lieu suivant leur sphère de circulation, privée ou publique (documents « pour soi », « pour autrui »), et selon leur proximité à des fonctions déjà matérialisées et formatées sur des supports papier (post-it, agenda, fax, formulaire...). De fait, les supports formatent les documents et leurs usages mais ne les déterminent pas de façon univoque, un même support pouvant avoir plusieurs usages comme la «feuille volante » ou l'« internet-intranet». Les usages s'inscrivent ainsi dans une " généalogie des usages ${ }^{7}$ » (Jouët, 2000) qui n'exclut pas leur évolution ou l'apparition d'usages innovants. De ce point de vue, à l'heure actuelle, les deux types d'usage papier et électronique semblent coexister avec un élargissement de la palette des choix offerts. Par exemple, une même personne peut utiliser simultanément un agenda papier et un agenda électronique, ce qui pose le problème de la synchronisation des deux agendas; de même, des agendas papier individuels peuvent coexister avec un agenda collectif électronique. Au final, l'apparition de nouveaux supports aurait plutôt tendance à exacerber l'importance des compétences générales, notamment de lecture/écriture.

L'enquête exploratoire avait également mis en évidence l'importance de la question du temps dans les activités de lecture et d'écriture. Nous avons pu ainsi saisir l'opportunité d'approfondir ce thème dans une enquête statistique portant sur les effets des TIC sur les temps sociaux : rythmes, durée et organisation du travail.

\section{LA LECTURE-ÉCRITURE : UNE ACTIVITÉ ESSENTIELLE CHEZ LES «SUROCCUPÉS »}

Suite à l'enquête exploratoire montrant l'importance actuelle des activités de lecture et d'écriture au travail, et leur report fréquent après le travail au-delà des horaires contractuels (Moatty, Rouard, Teiger, 2004), des questions concernant ces activités ont été introduites dans une enquête réalisée pour l'Institut Chronopost en 2004 (Tempos, 2005). Cette enquête portait sur les « Temps sociaux et les technologies de l'information et de la communication (TIC) »; elle s'est focalisée sur la population des « suroccupés »,

\footnotetext{
${ }^{7}$ La notion de généalogie des usages renvoie au fait que les nouveaux usages, notamment ceux des TIC, s'appuient sur les usages antérieurs.
} 


\section{Encadré 3 \\ Les suroccupés et leurs caractéristiques}

L'enquête téléphonique 2004 de l'Institut Chronopost, réalisée par lpsos auprès de 1004 actifs occupés issus d'un échantillon représentatif de la population française âgée de 15 ans et plus, étudie des activités de lecture et d'écriture effectuées au-delà des temps contractuels (Revue Tempos, 2005). Elle ne permet pas d'étudier les autres activités effectuées durant ces dépassements, ni les activités de lecture et d'écriture durant les temps contractuels. Elle mesure les temps de travail contractuels et effectifs individuels à partir des déclarations des enquêtés à trois questions : durée hebdomadaire contractuelle de travail, dépassements des horaires contractuels et volume moyen hebdomadaire de ces dépassements, qu'ils soient ou non rémunérés. Cette mesure diffère en partie de celle adoptée dans l'Enquête Emploi de l'Insee qui mesure les nombres d'heures effectuées par semaine puis normalement prévues, avant d'interroger sur les heures supplémentaires (ou complémentaires), rémunérées ou non, d'une semaine de référence.

Le questionnaire prévoyait le cas des enquêtés n'ayant pas de durée contractuelle de travail, ce qui est souvent le cas des artisans, commerçants, chefs d'entreprise, agriculteurs, mais la question " II vous arrive d'effectuer des tâches liées à votre activité professionnelle en plus de votre horaire de travail contractuel " a été posée à tous, le taux de "ne sait pas » étant seulement de 2,3\%. Les enquêtés appartenant aux catégories professionnelles citées ont répondu à la question des dépassements horaires, même lorsqu'ils n'avaient pas d'horaire contractuel. Le taux de non-réponse est cependant plus élevé, $8 \%$, car la question n'est pas adaptée à leur cas. Ils ont vraisemblablement répondu en fonction de leurs horaires effectifs de travail dont ils estiment qu'ils dépassent généralement les normes contractuelles nationales en cours (39 ou 35 heures). Nous désignerons ces deux cas à travers l'expression de "dépassements horaires".

Après la mise en place des 35 heures, l'écart entre les durées contractuelle et effective du travail reste important. En 2004, 43 \% des enquêtés sont concernés par les dépassements horaires, même occasionnels, qui atteignent en moyenne 5,8 heures par semaine. II s'agit d'une population plutôt qualifiée et plutôt diplômée.

dont la durée effective de travail dépasse le temps de travail contractuel. Dans ce cadre, nous avons pu introduire des questions, issues de l'enquête exploratoire, sur la pratique de cinq activités de lecture et d'écriture: rédaction de documents, relecture et correction de documents, les activités de courrier, lecture de documents papier, enfin la recherche d'informations professionnelles. Nous avons donc pu étudier les activités de lecture et d'écriture des suroccupés et les outils TIC utilisés durant les heures additionnelles (encadré 3). Notre propos était de mesurer l'importance de ces activités, d'en analyser les conditions de réalisation, et enfin d'identifier les motivations des enquêtés à les reporter (Moatty, Rouard, Teiger, 2006).

L'enquête exploratoire a montré l'importance des activités de lecture et d'écriture au travail et les contraintes temporelles qui y sont associées. Nous avons fait l'hypothèse qu'elles étaient centrales dans le hors travail chez les suroccupés lorsqu'ils travaillent au-delà de leurs horaires contractuels. Ils sont en effet $94 \%$ à déclarer au moins une activité de lecture ou d'écriture lors de leurs dépassements horaires. L'activité la plus fréquente est la lecture de documents papier (85\%), suivie de la recherche d'informations professionnelles ou de documentation (79\%). L'activité d'écriture est également très fréquente, la plus citée est la rédaction de documents (79\%), suivie de l'écriture de courriers ou de messages professionnels $(72 \%)$. Enfin, « relire et corriger des documents produits par une autre personne », c'est-à-dire l'activité de validation, est moins fréquente et concerne $54 \%$ des suroccupés ${ }^{8}$.

${ }^{8}$ L'enquête ne permet pas d'évaluer la durée de ces différentes activités. 


\section{Des inégalités de formation} et de position professionnelle

Pour comprendre les dépassements horaires des suroccupés en termes de lecture et d'écriture au travail, il est nécessaire de s'intéresser à leur formation et leur situation professionnelle.

\section{À la base, les acquis scolaires}

Les activités de lecture et d'écriture au travail mettent en œuvre des compétences générales et professionnelles. Si elles sont pratiquées à tout âge de manière assez homogène chez les actifs suroccupés, des clivages apparaissent selon le niveau scolaire (tableau 1). Moins fréquentes en dessous du niveau BEPC (Brevet d'études du premier cycle), elles augmentent avec le niveau de diplôme, même si la lecture reste moins clivante que l'écriture.

Ces résultats s'interprètent en termes de degré d'acquisition scolaire. Ainsi, la rédaction, qui suppose notamment de structurer l'écrit en termes de plan, de maîtriser la langue écrite et d'élaborer le contenu et le style du document, fait appel à des compétences scolaires acquises au niveau du lycée. Il en va de même pour celles mobilisées en lecture pour la recherche documentaire. Le courrier présente une hiérarchie plus marquée entre les différents niveaux scolaires car sa pratique suppose des relations professionnelles dont l'accroissement va de pair avec les diplômes : les plus diplômés ont des activités plus qualifiées, plus complexes, qui nécessitent des relations avec des collègues ou des partenaires extérieurs. On constate de plus qu'ils occupent une place particulière les amenant à déléguer les tâches d'écriture : s'ils rédigent moins, ils ont en revanche de fortes responsabilités de correction et de validation pour les écrits rédigés par d'autres personnes.

Le niveau des bacheliers est proche de la moyenne, pour l'ensemble des activités, à l'exception du courrier. Or, chez les suroccupés, deux tiers des titulaires du niveau BEPC rédigent des documents (64\%), ce qui signifie que les activités d'écriture et de lecture s'étendent à des professionnels peu formés en ce domaine. De plus, contrairement aux idées reçues, l'écriture professionnelle ne se réduit pas à la rédaction. En effet, un document technique doit pouvoir être compris par ses utilisateurs. Les bons écrits professionnels, adaptés à leur public, requièrent des

Tableau 1

Lecture et écriture professionnelles pendant les dépassements horaires chez les suroccupés selon les niveaux scolaires (en \%)

\begin{tabular}{|l|c|c|c|c|c|}
\hline & $\begin{array}{c}\text { BEPC ou } \\
\text { moins }\end{array}$ & $\begin{array}{c}\text { Bac- } \\
\text { calauréat }\end{array}$ & $\begin{array}{c}\text { DEUG/BTS/ } \\
\text { DUT/Licence/ } \\
\text { Maîtrise }\end{array}$ & $\begin{array}{c}\text { DEA/DESS/ } \\
\text { Doctorat/ } \\
\text { Grande école }\end{array}$ & Ensemble \\
\hline $\begin{array}{l}- \text { Rédiger des documents (rapports, } \\
\text { comptes-rendus, notes... }\end{array}$ & 64 & 78 & 89 & 82 & 79 \\
\hline $\begin{array}{l}- \text { Relire et corriger des documents } \\
\text { produits par une autre personne }\end{array}$ & 44 & 53 & 57 & 73 & 54 \\
\hline $\begin{array}{l}- \text { Écrire ou répondre à des courriers } \\
\text { ou des messages professionnels }\end{array}$ & 57 & 65 & 81 & 90 & 72 \\
\hline - Lire des documents papier & 74 & 84 & 89 & 99 & 85 \\
\hline $\begin{array}{l}- \text { Rechercher des informations profes- } \\
\text { sionnelles ou se documenter }\end{array}$ & 62 & 76 & 88 & 96 & 79 \\
\hline
\end{tabular}

Source : Enquête téléphonique 2004 de l'Institut Chronopost, réalisée par Ipsos auprès de 1004 actifs occupés.

Champ : Actifs déclarant travailler au-delà de leurs horaires contractuels ou ayant des dépassements horaires $(\mathrm{n}=429)$.

Lecture : Parmi les actifs déclarant travailler au-delà de leurs horaires, $64 \%$ de ceux qui ont un niveau égal ou inférieur au BEPC rédigent des documents (rapports, comptes-rendus, notes...).

Les différences sont statistiquement significatives selon le test du Khi 2, significatif au seuil de $5 \%$ au moins.

Sigles : BEPC (Brevet d'études du premier cycle), DEUG (Diplôme d'études universitaires générales), BTS (Brevet de technicien supérieur), DUT (Diplôme universitaire de technologie), DEA (Diplôme d'études approfondies), DESS (Diplôme d'études supérieures spécialisées). 
connaissances spécifiques liées au secteur d'activité et au métier, à la structure de l'entreprise et à sa culture de l'écrit, notamment autour des règles de circulation. Le rédacteur doit savoir décliner des niveaux différents de synthèse, les cibler pour des interlocuteurs variés, et gérer les contraintes de mise en circulation et de confidentialité. La culture générale produite par l'école ne suffit donc pas pour maitriser la culture professionnelle de l'écrit que l'on peut qualifier de culture générale professionnelle. La maîtrise de l'écrit, c'est-à-dire de ses enjeux, s'acquiert en partie sur le tas. De nombreux actifs, à l'exception des cadres destinataires des stages de lecture rapide, n'ont pas de formation à ces écrits spécifiques. L'absence de formations initiale et continue entraîne des difficultés, des risques et une insécurité linguistique.

\section{Des clivages socioprofessionnels, notamment pour l'écriture}

Si l'écriture et la lecture se sont répandues au travail, elles restent liées à la situation professionnelle, l'écriture étant plus clivante que la lecture. La part des suroccupés effectuant ces activités s'accroît fortement avec les qualifications professionnelles (tableau 2). Elle augmente globalement selon que l'on est ouvrier ou employé ou que l'on possède une qualification plus élevée (profession intermédiaire, cadre ou profession libérale). Ce résultat précise l'opposition entre l'univers ouvrier et celui des salariés les plus qualifiés du point de vue de la transmission orale ou écrite des instructions (Moatty, 1995). Les artisans, commerçants, chefs d'entreprise sont très concernés par toutes ces activités, de même que les agriculteurs.

\section{- L'exemple du courrier}

Écrire ou répondre à des courriers ou des messages professionnels, quel qu'en soit le support papier ou électronique, présente les écarts les plus importants chez les suroccupés suivant les professions. Le courrier suppose une activité relationnelle avec des collègues, des services de l'entreprise, des fournisseurs ou des clients. Si presque tous les artisanscommerçants-chefs d'entreprise ou agriculteurs

Tableau 2

Lecture et écriture professionnelles pendant les dépassements horaires chez les suroccupés selon les situations professionnelles (en \%)

\begin{tabular}{|l|c|c|c|c|c|c|}
\hline & Ouvrier & Employé & $\begin{array}{c}\text { Profession } \\
\text { intermédiaire }\end{array}$ & $\begin{array}{c}\text { Cadre } \\
\text { supérieur, } \\
\text { profession } \\
\text { libérale }\end{array}$ & $\begin{array}{c}\text { Artisan, } \\
\text { commerçant, } \\
\text { chef } \\
\text { d'entreprise, } \\
\text { agriculteur }\end{array}$ & Ensemble \\
\hline $\begin{array}{l}\text { Au moins une activité de lecture ou } \\
\text { d'écriture }\end{array}$ & 82 & 87 & 98 & 98 & 100 & 94 \\
\hline $\begin{array}{l}- \text { Rédiger des documents (rapports, } \\
\text { comptes-rendus, notes...) }\end{array}$ & 49 & 70 & 90 & 83 & 92 & 79 \\
\hline $\begin{array}{l}- \text { Relire et corriger des documents } \\
\text { produits par une autre personne }\end{array}$ & 30 & 50 & 58 & 71 & 58 & 54 \\
\hline $\begin{array}{l}- \text { Écrire ou répondre à des courriers } \\
\text { ou des messages professionnels }\end{array}$ & 40 & 54 & 76 & 89 & 100 & 72 \\
\hline- Lire des documents papier & 65 & 78 & 91 & 91 & 92 & 85 \\
\hline $\begin{array}{l}- \text { Rechercher des informations pro- } \\
\text { fessionnelles ou se documenter }\end{array}$ & 54 & 63 & 88 & 94 & 99 & 79 \\
\hline
\end{tabular}

Source : Enquête téléphonique 2004 de l'Institut Chronopost, réalisée par Ipsos auprès de 1004 actifs occupés.

Champ : Actifs déclarant travailler au-delà de leurs horaires contractuels ou ayant des dépassements horaires $(\mathrm{n}=429)$.

Lecture : Parmi les actifs déclarant travailler au-delà de leurs horaires, $82 \%$ des ouvriers ont au moins une activité de lecture ou d'écriture. Les différences sont statistiquement significatives selon le test du Khi 2, significatif au seuil de $5 \%$ au moins. 
rédigent des courriers, seulement quatre ouvriers sur dix le font. Alors que la correspondance privée est considérée comme une forme d'écriture répandue dans tous les milieux, la rédaction de courrier à visée professionnelle apparait très différente et reflète les hiérarchies socioprofessionnelles.

\section{Lecture-écriture à l'ère électronique}

À l'origine, l'écriture a été une activité technique réservée à des spécialistes. Aujourd'hui, l'ordinateur favorise la prolifération des écrits dans l'univers du travail. Mais l'ordinateur crée rarement des usages ex nihilo ; il vient généralement équiper des pratiques déjà existantes en s'inscrivant dans une généalogie des usages. Tisser un rapport à l'écrit est déterminant dans le recours à l'ordinateur. Les suroccupés qui déclarent n'effectuer aucune des tâches de lecture ou d'écriture utilisent moins souvent des ordinateurs que ceux qui en déclarent une ou plus : le taux de personnes équipées s'accroît ainsi de $28 \%$ à $73 \%$. Tisser un rapport à l'écrit est donc bien déterminant dans le recours à l'ordinateur. Utiliser des équipements informatiques favorise les activités d'écriture. Ceux qui n'ont pas d'ordinateur professionnel ou personnel ne sont que $60 \%$ à rédiger des documents, alors qu'ils sont $87 \%$ lorsqu'ils disposent d'un ordinateur fixe et $93 \%$ lorsqu'il s'agit d'un portable. L'activité de courrier présente une hiérarchie assez similaire.

L'activité d'écriture corrective, qui relève du contrôle hiérarchique ou de l'expertise, clive fortement les suroccupés et confirme le lien entre niveau d'équipement et niveau de fonction et de qualification. Seulement $36 \%$ des suroccupés ne disposant pas d'ordinateurs ont à valider des documents, alors qu'ils sont $59 \%$ lorsqu'ils ont un ordinateur fixe et $68 \%$ lorsqu'il s'agit d'un portable.

Les activités de lecture varient selon que l'on dispose ou non d'un ordinateur. Ainsi, l'écart entre l'absence d'ordinateur et la possession d'un portable est beaucoup plus important pour la recherche d'informations professionnelles (42\%) que pour la lecture de documents papier (23\%). L'ordinateur apparait comme un support très utile dans l'activité de documentation professionnelle.

Si l'ordinateur vient équiper les pratiques d'écriture et de lecture, rappelons que l'analyse de cet effet de l'ordinateur doit être pondérée par la prise en compte d'un biais de sélection, l'attribution d'un ordinateur étant plus fréquente pour les diplômés ou les qualifiés.

\section{Une reconnaissance inégale des écrits par l'organisation}

L'intensité des pratiques varie selon les secteurs d'activité des suroccupés. Le monde de la production matérielle entretient un rapport à l'écrit plus distendu que celui des bureaux. Par exemple, lors des dépassements horaires, la rédaction de documents concerne moins les salariés de l'industrie $(67 \%)$ ou de la construction $(60 \%)$ que ceux des services $(85 \%)$ ou des administrations (89\%). Mais, comme l'a montré l'enquête exploratoire, la prolifération des activités d'écriture au travail n'est pas toujours pensée par l'organisation ni prévue de la même façon dans ces deux univers. L'univers des bureaux est souvent associé à un monde de papiers ou d'écritures. Dans le monde industriel, les activités de lecture et d'écriture ne sont pas toujours prévues dans l'emploi du temps, même si elles peuvent être décrites dans certaines fiches de postes. Ces activités invisibles et peu reconnues coûtent d'autant plus aux individus qu'elles correspondent à des lacunes organisationnelles qu'ils doivent pallier, au détriment de leur temps personnel.

\section{Des dépassements horaires liés aux conditions de travail inadaptées}

Pour $80 \%$ des suroccupés, les dépassements horaires sont une nécessité liée au métier ou au poste, par exemple à la charge de travail. Ces dépassements relèvent le plus souvent, pour $62 \%$ des enquêtés, de leur propre initiative plutôt que d'une demande de l'employeur. En contrepartie, les suroccupés ont plus fréquemment la possibilité formelle ou informelle de travailler à domicile. Cette population, plutôt qualifiée et flexible tant du point de vue du temps et des lieux de travail que de la séparation entre espaces privé et professionnel, est davantage équipée de TIC (ordinateurs fixes et portables, téléphone portable...).

La lecture et l'écriture se poursuivent au-delà des horaires contractuels, le plus souvent en restant sur le lieu de travail (69\%), parfois au domicile (27\%), et plus rarement (4\%) dans les transports ou dans un autre lieu, contrairement à l'idée reçue d'un report à 
la maison en cas de surcharge de travail. Ce report de l'activité de travail au domicile est perçu comme moins contraignant car pouvant se concilier avec des contraintes d'ordre privé. L'écriture est réalisée de manière privilégiée sur le lieu de travail tandis que la lecture, bien qu'y restant le plus souvent effectuée, se fait également à domicile. Ainsi ceux qui rédigent des documents au-delà de leurs horaires le font à $65 \%$ au travail et à $35 \%$ au domicile, tandis que ceux qui lisent des documents papier le font à $54 \%$ au travail, à $44 \%$ au domicile et enfin à $2 \%$ dans les transports.

Les motifs avancés pour travailler au domicile sont liés à la qualité des conditions de travail pour des activités de lecture ou d'écriture. Travailler à domicile permet de choisir plus librement le moment propice pour s'y consacrer ( $45 \%$ ), de pouvoir travailler sans être dérangé ( $43 \%$ ) ou d'achever une tâche que l'on a pas eu le temps de réaliser durant les horaires contractuels parce que l'on travaille dans l'urgence (40\%). Il s'agit plus rarement d'organiser la journée de travail en fonction des contraintes personnelles (18\%). Le travail au domicile est donc davantage lié à la poursuite de l'activité professionnelle qu'à la conciliation entre vie privée et vie professionnelle.

Les raisons citées pour rester sur le lieu de travail lors des dépassements horaires sont principalement liées au souhait de séparer vies privée et professionnelle (37\%), mais aussi aux besoins d'échanges avec les collègues ou le supérieur hiérarchique (33\%). Ensuite viennent des raisons matérielles, absence au domicile de ressources (banques de données, documentation) (26\%) ou de l'équipement nécessaire (ordinateur, logiciels) (23\%). De plus, pour une fraction des suroccupés se pose la question du droit de sortir des documents (15\%). Par ailleurs, il existe un vide juridique, les contrats de travail ne prévoient pas le report du travail à la maison.

Tout se passe comme si les tâches longues, complexes, ou nécessitant un équipement et des ressources disponibles sur le lieu de travail y restent localisées, tandis que des tâches plus ponctuelles ou moins perçues comme du travail s'effectuent plus volontiers à la maison.

De bonnes conditions de travail pour les activités d'écriture et de lecture ne sont donc pas toujours réunies au travail. La prise en compte par l'organisation de ces activités améliorerait le travail et permettrait aux professionnels de disposer de lieux et de moments pour se concentrer sur ces activités et de les effectuer sur leurs temps contractuels. Ces résultats montrent que les activités de lecture et d'écriture, loin d'être des activités immatérielles, sont des activités qui nécessitent des conditions de travail adaptées. La reconnaissance de ces activités comme du travail, dans tous les métiers, permettrait de mieux prévoir les temps de réalisation et d'identifier les compétences et les formations nécessaires. Le développement de compétences est donc nécessaire mais pas suffisant et au-delà de la formation, il faut prendre en compte les conditions de travail des activités intellectuelles dans l'organisation du travail.

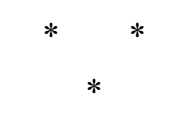

Les résultats présentés ici précisent la place et l'importance du langage au travail à travers la prolifération des activités liées à l'écrit, l'écriture mais aussi la lecture professionnelles. Les enjeux de formation sont liés aux trois types de compétences langagières à l'œuvre au travail, compétences générales (linguistiques et techniques), compétences culturelles et compétences sociales (dans leur dimension relationnelle).

L'enquête qualitative préalable auprès de techniciens et d'ingénieurs en formation au CNAM a permis d'inventorier les activités de lecture/écriture papier ou électroniques et d'en préciser les propriétés, leur variété, l'intrication de la lecture et de l'écriture et l'imbrication de l'écrit avec l'oral. Elle a montré l'importance des conditions de leur réalisation, notamment temporelles, en raison de leur caractère chronophage et du manque de temps alloué pour les réaliser, ce qui entraîne leur report après le travail. Elle a identifié des compétences de l'écrit : synthèse, style et manières de dire, choix d'un point de vue. Elle a révélé des apprentissages par essais et erreurs induisant des risques et une insécurité linguistique chez les individus qui peuvent être humiliés voire sanctionnés en raison d'un manquement aux normes. Elle a montré l'importance des compétences générales, culturelles et sociales pour la maîtrise de l'écrit 
(règles de circulation des écrits " pour soi » et des écrits « pour autrui »), relativement aux compétences techniques liées aux supports électroniques.

Les résultats quantitatifs sur les suroccupés montrent l'importance des dépassements horaires et la place essentielle qu'y tiennent les activités de lecture et d'écriture. Ils signalent des lacunes dans la formation à ces activités, la sous-estimation par l'organisation $\mathrm{du}$ travail qu'elles représentent et le report sur les individus de contraintes liées au travail. De plus, les conditions de travail ne sont pas toujours réunies pour leur réalisation. Tous ces facteurs contribuent à maintenir, voire à accentuer, les disparités selon les niveaux scolaires ou la situation professionnelle.
En conclusion, les résultats soulignent l'importance et la spécificité de la culture écrite dans le monde du travail. Ils montrent que la question de la formation des salariés aux compétences langagières au travail concerne tous les salariés, quel que soit leur niveau de qualification, et ne se réduit pas seulement à un problème de contenus de formation; elle nécessite aussi de prendre en compte les conditions du travail intellectuel dans les entreprises. Les activités de lecture et d'écriture sont un travail à part entière qui doit être intégré dans l'organisation du travail en termes de temps et de lieu de réalisation, de modalités d'apprentissage et d'appropriation avec la reconnaissance des trois types de compétences nécessaires.

\section{Bibliographie}

Bailly F., Blanc M. Dezalay T., Peyrard C. (2002), Pratiques professionnelles et usages des écrits électroniques, L'Harmattan, Paris, 236 p.

Borzeix A., Fraenkel B. (coord.) (2001), Langage et Travail, CNRS, Paris, 384 p.

Boutet J. (1995), Paroles au travail, L'Harmattan, Paris, pp. 247-267.

Boutet J. (2008), La vie verbale au travail, Octarès, Toulouse.

Boutet J., Gardin B. (2001), «Une linguistique du travail », in Borzeix A., Fraenkel B., Langage et Travail, CNRS, Paris, pp. 89-113.

Chartier R. (Dir.) (1992), La correspondance. Les usages de la lettre au XIXe siècle, Fayard, Paris.

Christin A.-M. (Dir.) (2001), Histoire de l'écriture, Seuil, Paris.

Cochoy F., Garel J.-P., Terssac G. (1998), « Comment l'écrit travaille l'organisation: le cas des normes
ISO $9000 »$, Revue française de sociologie, vol. XXXIX-4, pp. 673-699.

Dugué E. (1994), « La gestion des compétences : Les savoirs dévalués, le pouvoir occulté », Sociologie $d u$ travail, Dunod, pp. 273-292.

Fraenkel B. (1995), «La traçabilité une fonction caractéristique des écrits de travail », in Dire et faire au travail, Connexions, Erès, n 65/1, pp. 63-76.

Girin J. (1995), « Les agencements organisationnels », in Charue-Duboc F. (dir.), Des savoirs en action. Contribution à la recherche en gestion, L'Harmattan, Paris, pp. 233-279.

Grignon C. (1976), «L'art et le métier, école parallèle et petite bourgeoisie ", Actes de la Recherche en Sciences Sociales, 4.

Grosjean M., Lacoste M. (1998), «L'oral et l'écrit dans les communications de travail ou les illusions du "tout écrit" ", Sociologie du Travail, Dunod, vol. XL, n 4, pp. 439-461. 
INRP/CNAM/CNRS-LT, Lazar A. (coord.) (1998), Langage(s) et travail : enjeux de formation, Actes du Colloque INRP/CNAM/CNRS-LT, Paris, 13-15 octobre, $516 \mathrm{p}$.

Institut Chronopost, (2005), « Temps sociaux, les TIC changent-elles la donne?», Revue Tempos, $\mathrm{n}^{\circ} 3$, janvier, $64 \mathrm{p}$.

Jouët J. (2000), « Retour critique sur la sociologie des usages », Réseaux, n 100, pp. 487-521.

Moatty F. (1995), «Les communications de travail : comment et avec qui ? », Centre d'études de l'emploi, Noisy-le-Grand, La Lettre, ${ }^{\circ} 39$, octobre, $10 \mathrm{p}$.

Moatty F. (2000), « Instructions de travail écrites et bas niveaux de qualification », in C. El Hayek (coord.), Illettrisme et monde du travail, Paris, La Documentation française/MES-GPLI, Paris, pp. 61-74.

Moatty F., Rouard F., Teiger C. (2004), « Les temps de l'écrit dans les nouvelles formes d'organisation du travail », in collectif GRIOT, Figures du temps - Les nouvelles temporalités du travail et de la formation, L'Harmattan, Paris, pp. 149-167.

Moatty F., Rouard F., Teiger C. (2005), « Montée de l'écrit au travail et transformation de l'évaluation ", in M.-C. Bureau et E. Marchal (Eds.), Au risque de l'évaluation. Salariés et candidats à l'emploi soumis aux aléas du jugement, Presses Universitaires du Septentrion, Lille, pp. 175-201.

Moatty F., Rouard F., Teiger C. (2006), « Le travail au-delà du travail : lire et écrire au-delà des temps contractuels », La Découverte, Paris, pp. 85-94.

Moatty F., Rouard F., Teiger C. (2007), « Lectures pour soi, lectures invisibles? Une cartographie des lectures au début de l'internet », in Saint LaurentKogan A.-F. et Metzger J.-L. (Dir.), Où va le travail à l'ère du numérique, Presses de l'École des Mines de Paris, Paris, pp. 197-210.
Pène S., Borzeix A., Fraenkel B. (coord.) (2001), Le langage dans les organisations, L'Harmattan, Paris, $244 \mathrm{p}$.

Pommier P., Zamora P. (2008), «La formation continue : un objet de négociation au confluent des stratégies des entreprises et des besoins des salariés ? », Premières Synthèses, DARES, n 14.2, $8 \mathrm{p}$.

Pottier F. (1996), «Les ingénieurs du CNAM : quel devenir? », Formation Emploi, n ${ }^{\circ}$ 55, pp. 59-75.

Rouard F. (1998), «Écriture et normes : l'exemple des étudiants en communication du CNAM », in A. Lazar (coord.), Langage(s) et travail : enjeux de formation, INRP/CNAM/CNRS-LT, Paris, pp. 64-70.

Rouard F. (1995), «L'individu et ses traces dans les écrits au travail», Le Gré des langues, $\mathrm{n}^{\circ}$ 9, L'Harmattan, Paris, pp. 152-175.

Rouard F. (2000), «Écritures au travail et insécurité linguistique », in $\mathrm{C}$. El Hayek (coord.), Illettrisme et monde du travail, La Documentation française/MESGPLI, Paris, pp. 53-60.

Teiger C. (1995), «Parler quand même : les fonctions des activités langagières non fonctionnelles », in J. Boutet (Dir.), Paroles au travail, L'Harmattan, Paris, pp. 45-72.

Teiger C., Rouard F., Moatty F. (2000), « La dimension langagière de la culture technique dans le travail », étude pour la Délégation générale à la Langue française, Paris, novembre, $53 \mathrm{p}$.

Teiger C., Rouard F., Moatty F. (2002), La culture des techniciens: les pratiques d'écriture au travail, rapport final pour le ministère de la Culture et de la Communication, CNAM (Laboratoire d'Ergonomie) CEE, 31 mai, $277 \mathrm{p}$. 


\section{Résumé}

\section{Lecture et écriture au travail : \\ les enjeux en termes de formation et de conditions de travail}

Frédéric Moatty et Françoise Rouard

L'article montre l'importance des activités de lecture et d'écriture au travail et les problèmes de formation des salariés inhérents à ces activités. II rappelle les recherches sur la place et l'importance du langage au travail et s'intéresse aux compétences langagières, de lecture et d'écriture notamment. II présente ensuite les catégories d'analyse des activités langagières élaborées lors d'une enquête qualitative auprès de techniciens et d'ingénieurs en formation au CNAM (Conservatoire national des arts et métiers). II étudie ces activités grâce à une enquête menée auprès de salariés dont la durée effective de travail dépasse la durée de travail contractuelle. II conclut sur les enjeux en termes de formation des salariés mais aussi de conditions du travail intellectuel dans les entreprises.

\section{Mots clés}

Conditions de travail, formation professionnelle, compétence, information-communication Journal of Economic Literature: M 53, J 81 